\title{
Differential Short-Term Plasticity at Convergent Inhibitory Synapses to the Substantia Nigra Pars Reticulata
}

\author{
William M. Connelly, ${ }^{1,2}$ Jan M. Schulz, ${ }^{2}$ George Lees, ${ }^{1}$ and John N. J. Reynolds ${ }^{2}$ \\ Departments of ${ }^{1}$ Pharmacology and Toxicology and ${ }^{2}$ Anatomy and Structural Biology, School of Medical Sciences, University of Otago, Dunedin 9054, \\ New Zealand
}

\begin{abstract}
Inhibitory projections from the striatum and globus pallidus converge onto GABAergic projection neurons of the substantia nigra pars reticulata $(\mathrm{SNr})$. Based on existing structural and functional evidence, these pathways are likely to differentially regulate the firing of $\mathrm{SNr}$ neurons. We sought to investigate the functional differences in inhibitory striatonigral and pallidonigral traffic using whole-cell voltage clamp in brain slices with these pathways preserved. We found that striatonigral IPSCs exhibited a high degree of paired-pulse facilitation. We tracked this facilitation over development and found the facilitation as the animal aged, but stabilized by postnatal day 17 (P17), with a paired pulse ratio of 2 . We also found that the recovery from facilitation accelerated over development, again, reaching a stable phenotype by P17. In contrast, pallidonigral synapses show paired-pulse depression, and this depression could be solely explained by presynaptic changes. The mean paired-pulse ratio of 0.67 did not change over development, but the recovery from depression slowed over development. Pallidonigral IPSCs were significantly faster than striatonigral IPSCs when measured at the soma. Finally, under current clamp, prolonged bursts of striatal IPSPs were able to consistently silence the pacemaker activity of nigral neurons, whereas pallidal inputs depressed, allowing nigral neurons to reinstate firing. These findings highlight the importance of differential dynamics of neurotransmitter release in regulating the circuit behavior of the basal ganglia.
\end{abstract}

\section{Introduction}

The substantia nigra is a major subdivision of the basal ganglia and consists of the primarily dopaminergic substantia nigra pars compacta $(\mathrm{SNc})$ and the GABAergic substantia nigra pars reticulata $(\mathrm{SNr})$. The $\mathrm{SNr}$ is a major output nucleus for the basal ganglia and delivers inhibitory efferents to the relay nuclei of the thalamus. The $\mathrm{SNr}$ receives glutamatergic inputs from the subthalamic and pedunculopontine nuclei as well as the prefrontal cortex (Kita and Kitai, 1987; Lavoie and Parent, 1994; Naito and Kita, 1994). However, the majority of afferents to the $\mathrm{SNr}$ are inhibitory, arising from the globus pallidus (GP) and the striatum and to a lesser extent local axon collaterals from other SNr neurons (Ribak et al., 1980; Deniau et al., 1982; Araki et al., 1985; Smith and Bolam, 1991).

Pallidonigral and striatonigral fibers converge onto SNr neurons, but they synapse onto primarily separate domains of the somatodendritic tree. Pallidonigral terminals form large baskets around the somatic domain and proximal dendrites of SNr cells, whereas striatonigral synapses are formed on fine distal dendrites (Smith and Bolam, 1991; von Krosigk et al., 1992). Furthermore, the activity of neurons in the GP and striatum are different. Striatal medium spiny cells (that give rise to the striatonigral pathway) can be viewed as relatively silent compared with the highly

Received July 26, 2010; revised Aug. 24, 2010; accepted Aug. 29, 2010.

This work was supported by the Neurological Foundation of New Zealand (W.M.C., G.L.) and a grant from the Marsden Fund of the Royal Society of New Zealand (J.N.J.R.).

Correspondence should be addressed to William M. Connelly, Department of Anatomy and Structural Biology, University of Otago, P.0. Box 913, Dunedin 9054, New Zealand. E-mail: bill.connelly@otago.ac.nz.

DOI:10.1523/JNEUROSCI.3895-10.2010

Copyright $\odot 2010$ the authors $\quad 0270-6474 / 10 / 3014854-08 \$ 15.00 / 0$ active neurons of the GP. In awake animals, medium spiny neurons typically exhibit a low basal firing rate of $<5 \mathrm{~Hz}$ (typically $0.02-3 \mathrm{~Hz}$ ) but may show movement-associated bursts up to 100 $\mathrm{Hz}$ (Wilson and Groves, 1981; Ryan et al., 1989; Kimura, 1990; Kiyatkin and Rebec, 1996; Mahon et al., 2006). In contrast, pallidal neurons fire tonically at $50-70 \mathrm{~Hz}$ but regularly experience long (>500 ms) pauses in firing, and it appears that these burstpause complexes are movement related (DeLong, 1971; Raz et al., 2000).

Although the simplicity of classical rate coding is appealing when discussing the function of the basal ganglia, synapses show a variety of frequency-dependent processes (Zucker and Regehr, 2002). Therefore, we sought to investigate the frequency dependence of the striatonigral and the pallidonigral pathways. Because we could only prepare slices with these pathways intact from relatively juvenile animals, we also tracked how this frequency dependence changed over development. Here, we show that striatonigral medium spiny neurons in the so-called "direct" basal ganglia pathway form facilitating synapses onto GABAergic projection neurons of the SNr. In contrast, synapses from pallidonigral neurons, which receive input from spiny neurons of the "indirect" pathway, depress. Furthermore, we describe differences in the kinetics of the influence of these two converging systems on basal ganglia output and changes in these effects over development.

\section{Materials and Methods}

All procedures involving animals were approved by the University of Otago Animal Ethics Committee. Fourteen- to 20-d-old C57BL/6 mice pups were maintained on a $12 \mathrm{~h}$ light/dark cycle. Pups were rapidly decapitated, and brains were dissected out into ice-cold modified artifi- 
A
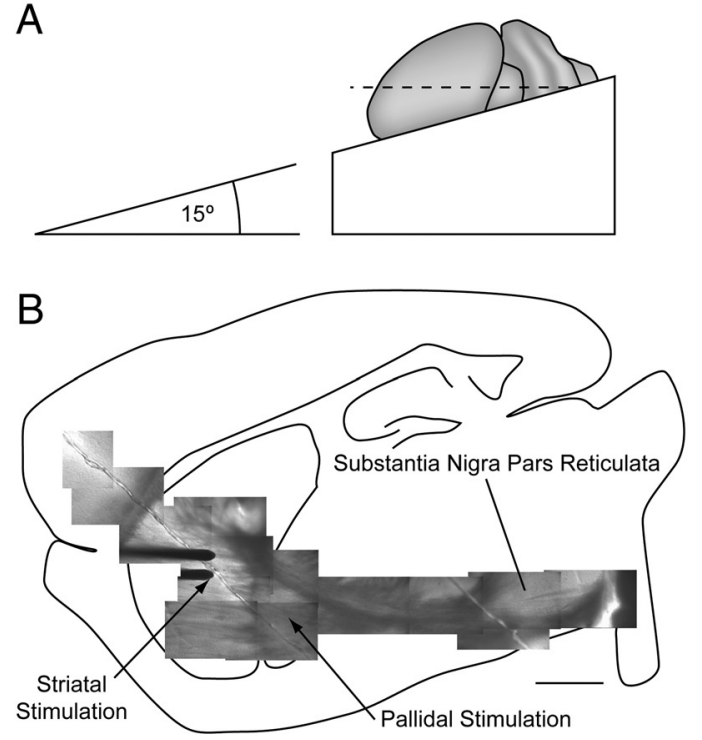

C

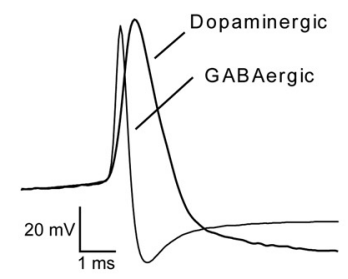

$\mathrm{D}$

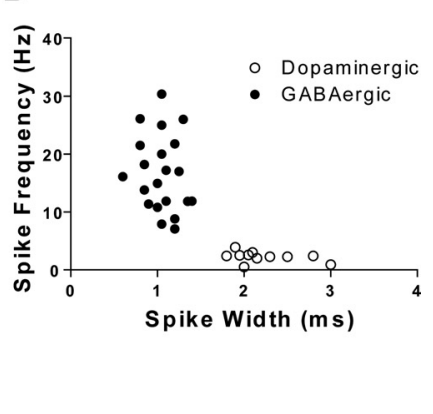

Figure 1. Slice orientation and physiology of SNr neurons. A, Parasagittal slices were taken at $15^{\circ}$ from the midline. Dotted line shows the approximate location of the slice. $\boldsymbol{B}$, Compound photomicrograph showing the position of the electrodes for striatal stimulation (electrode tips visible) and pallidal stimulation. Also shown is the location of the recording electrode in the $\mathrm{Nr}$ (patch electrode is faintly visible). Scale bar, $1 \mathrm{~mm}$. C, A typical action potential recorded from the GABAergic neurons of the SNr and the dopaminergic neurons of the SNc. D, Spike width and tonic spiking frequency plotted against each other showing the distinctive firing patterns of two classes of nigral neurons. All neurons with a GABAergic phenotype were recorded randomly from the $\mathrm{NNr}$. Dopaminergic neurons could only be recorded when pipettes were positioned in the SNC.
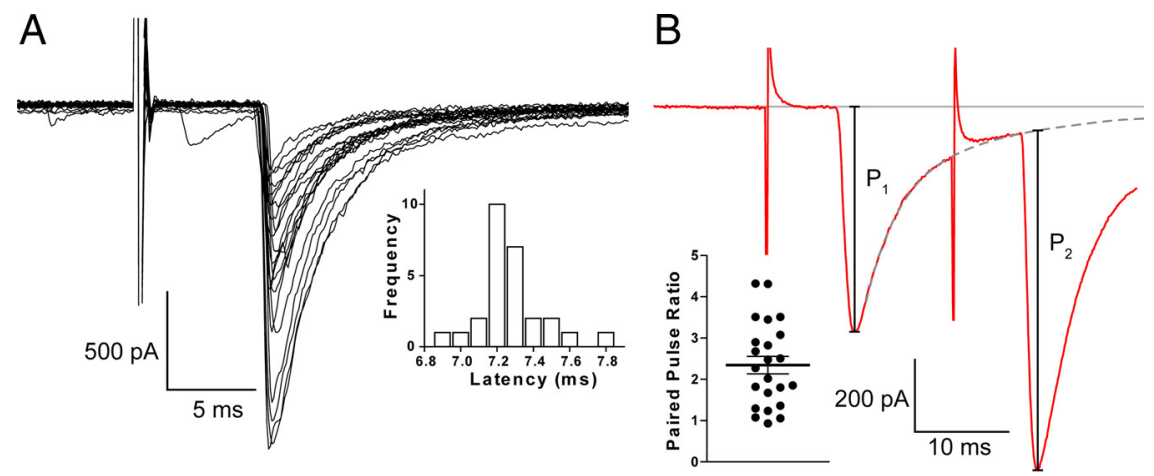

Figure 2. Baseline characteristics of striatonigral elPSCs. $A$, Overlay of numerous elPSCs induced by stimulating in the striatum and recording in the SNr. Inset, Distribution of elPSC latencies showing their tight distribution. $\boldsymbol{B}$, Striatonigral IPSCS facilitate. When an eIPSC was evoked during the decaying phase of the previous IPSC, a (bi)exponential was fit to the decay, and the amplitude of the second event was calculated relative to the extrapolated decay of the first. Inset, The paired-pulse ratio (P2/P1) at $50 \mathrm{~Hz}$ calculated across all ages.

cial CSF (aCSF) (in mm: 248 sucrose, $3 \mathrm{KCl}, 2 \mathrm{MgCl}_{2}, 1 \mathrm{CaCl}_{2}, 1.25$ $\mathrm{NaH}_{2} \mathrm{PO}_{4}, 26 \mathrm{NaHCO}_{3}$, and 10 glucose) that had been saturated with $95 \% \mathrm{O}_{2} / 5 \% \mathrm{CO}_{2}$. Three-hundred-micrometer-thick parasagittal slices were cut at $15^{\circ}$ to the midline, such that the caudal end of the slice was closer to the midline (see Fig. 1A) (Wallmichrath and Szabo, 2002; Beurrier et al., 2006). Slices were placed in a holding chamber at $35^{\circ} \mathrm{C}$ in aCSF (in mm: 124 $\mathrm{NaCl}, 3 \mathrm{KCl}, 1 \mathrm{MgCl}_{2}, 2 \mathrm{CaCl}_{2}, 1.25 \mathrm{NaH}_{2} \mathrm{PO}_{4}, 26 \mathrm{NaHCO}_{3}, 10$ glucose, $1 \mathrm{Na}$-pyruvate, and $3 \mathrm{Na}$-ascorbate) that had been bubbled with $95 \%$ $\mathrm{O}_{2} / 5 \% \mathrm{CO}_{2}$ before being cooled to room temperature after $0.5 \mathrm{~h}$. Slices were maintained under these conditions for $0.5 \mathrm{~h}$, then placed in a recording chamber on the stage of a differential interference contrast (DIC) microscope (E600FM DIC by Nikon or BX51W by Olympus), and perfused at $\sim 2$ $\mathrm{ml} / \mathrm{min}$ with aCSF maintained at $33-35^{\circ} \mathrm{C}$ (ATR temperature controller by Quest Scientific or TC2BIP by Cell MicroControl). Visually identified SNr neurons were patched with borosilicate pipettes (1-3 M $\Omega$ ) filled with one of the following solutions (in $\mathrm{mm}$ ): $125 \mathrm{~K}$-gluconate, $2.5 \mathrm{KCl}, 7 \mathrm{NaCl}, 10$ HEPES, 0.5 EGTA, 2 Mg-ATP, 0.2 Na-GTP, and 4 phosphocreatine, pH 7.35 for measurements of spiking activity. For measurements of IPSCs, the following solution was used: $120 \mathrm{CsCl}, 10 \mathrm{HEPES}, 10$ EGTA, $1 \mathrm{CaCl}_{2}, 1$ $\mathrm{MgCl}_{2}, 5$ QX-314 [2(triethylamino)- $N$-(2,6dimethylphenyl) acetamine], 2 Mg-ATP, 0.2 $\mathrm{Na}_{2} \mathrm{GTP}, 4$ phosphocreatine, $\mathrm{pH}$ 7.35. Bipolar stimulating electrodes (FHC) were placed in the striatum or globus pallidus (see Fig. $1 B$ ), and events were evoked by single stimulus pulses (100 $\mu$ s duration) delivered by a constantvoltage isolated stimulator (S88; Grass Instruments). For eliciting events from striatal fibers, stimulus intensity was set at the minimal level necessary to produce an IPSC without failures, usually two to three times the minimum intensity required to evoke a clear response $(\sim 10-50 \mathrm{~V})$. As the striatonigral pathway passes through the GP (Preston et al., 1980; Wu et al., 2000), minimal stimulation (2-10 V) was used to evoke pallidonigral IPSCs to avoid recruiting coincident striatonigral IPSCs. A minimal event could be clearly recognized as an all-or-none IPSC that was recruited when the stimulation intensity passed some intensity. If no all-or-none IPSC could be evoked (that is, a graded response was seen), then the slice was deemed not to contain the pallidonigral connection and was discarded. Generally, striatal and pallidal events were not recorded from the same cell because of the difficulty of making long-lived recordings with low series resistance; however, both events could usually be seen in the same cell. Series resistance was compensated $70-90 \%$ (at $3 \mathrm{kHz}$ ) and data excluded if series resistance increased above $15 \mathrm{M} \Omega$ or changed by $>5 \mathrm{M} \Omega$ during recording. Data were filtered at $3 \mathrm{kHz}$ and digitized at $10 \mathrm{kHz}$ (micro1401; Cambridge Electronics Design). Evoked IPSCs (eIPSCs) were elicited once every 60 s. eIPSC amplitudes were calculated from the average of more than three trials, and, when evoked during the decay of the previous eIPSC, amplitudes were calculated as the difference between the peak current and the extrapolated (bi)exponential decay of the previous eIPSC (see Fig. 2 B). Latency was measured as the delay from stimulus artifact to the $10 \%$ rise time of the eIPSC. Jitter was calculated as the SD of the latency over $>15$ trials. Paired-pulse ratios were generally not normally distributed, but, after log transformation, data became normal and could then be subjected to one-way and two-way ANOVA. Reversal potentials were calculated by nonlinear regression using the Goldman-Hodgkin-Katz flux equation (Hille, 2001). All recordings were made in the presence of CNQX $(10 \mu \mathrm{M})$ and D-AP-5 $(20 \mu \mathrm{M})$.

\section{Results}

Using whole-cell voltage clamp in parasagittal brain slices, we sought to record eIPSCs in the GABAergic projection neurons of the SNr. However, there are occasional cells in the SNr with electrophysiological characteristics of dopaminergic cells from the SNc (German and Manaye, 1993), such as a slow firing frequency $(<5 \mathrm{~Hz})$ and broad action potentials $(>2 \mathrm{~ms})$, whereas GABAergic neurons fire faster than $5 \mathrm{~Hz}$ and have narrow action potentials ( $<1.5 \mathrm{~ms}$ ) (Yung et al., 1991; Richards et al., 1997; Wallmichrath and Szabo, 2002). We recorded from 20 randomly selected $\mathrm{SNr}$ neurons but did not encounter a single neuron displaying the 
A $100 \mathrm{~Hz}$
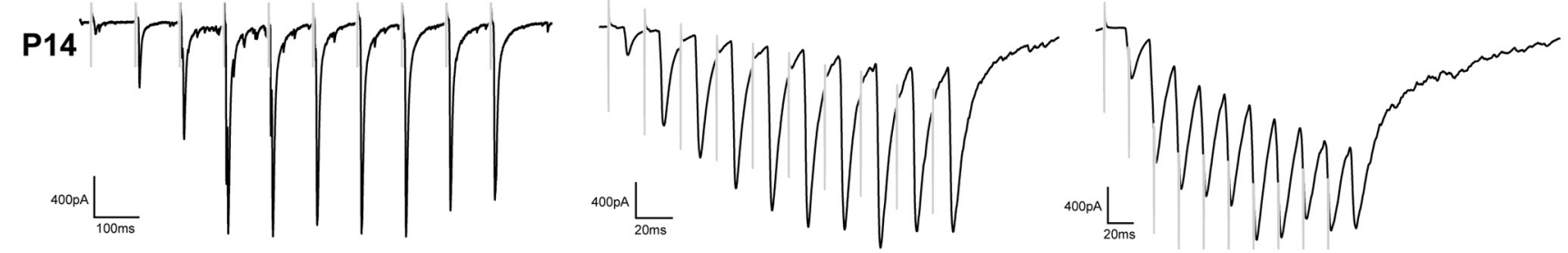

B
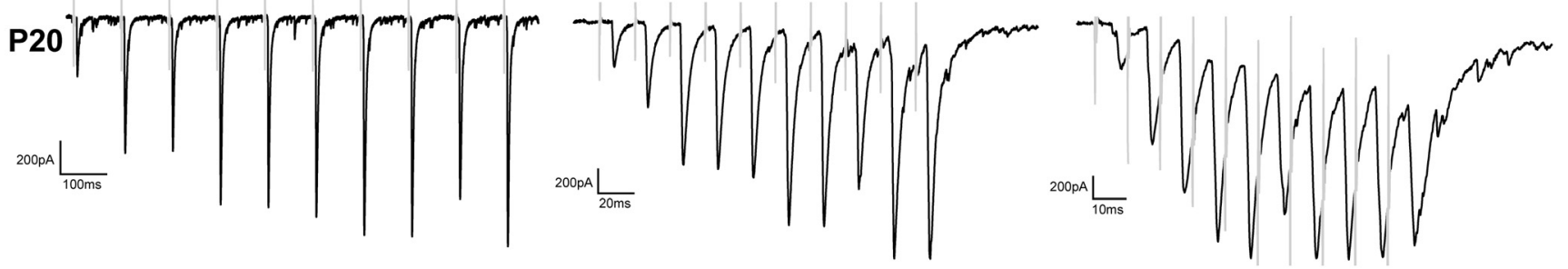

C
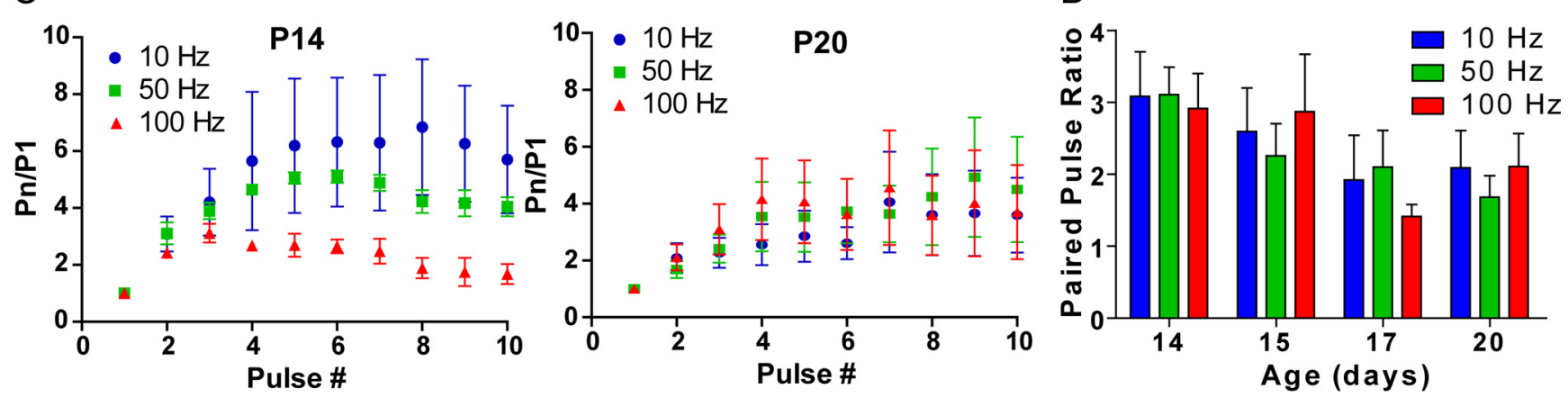

Figure 3. Striatonigral elPSCs undergo paired-pulse facilitation, which decreases with age. $\boldsymbol{A}, \boldsymbol{B}, \mathrm{A}$ train of 10 striatonigral elPSCs evoked at 10,50 , and $100 \mathrm{~Hz}$ recorded from a $\mathrm{P} 14(\boldsymbol{A})$ and a $\mathrm{P} 20$ $(\boldsymbol{B})$ animal. $\boldsymbol{C}$, Group statistics showing the large degree of facilitation seen in P14 $(n=3-6)$ animals and how this declines during high-frequency stimulation. P20 $(n=5-6)$ animals do not show the same degree of facilitation, and this does not decline at high frequencies. $\boldsymbol{D}$, The paired-pulse ratio between first and second pulse is unaffected by frequency in the $10-100 \mathrm{~Hz}$ range, but as animals age, the degree of facilitation decreases $(n=4-7 ; p=0.005)$. Stimulus artifacts are digitally truncated and shaded.

cellular properties of a dopaminergic neuron (Fig. 1C). Conversely, recordings from putative dopaminergic neurons were readily obtained in the easily discernible $\mathrm{SNc}$, but we did not encounter a single neuron with a GABAergic firing property (Fig. $1 D)$. Thereafter, we recorded from neurons in the SNr using a patch pipette solution that precluded the electrophysiological identification of neurons ( $\mathrm{CsCl}$ and QX-314), confident that our chance of accidentally recording from a dopaminergic neuron was $<1 / 20$.

Cells were held at $-70 \mathrm{mV}$ in aCSF at $35^{\circ} \mathrm{C}$ containing $\left[\mathrm{Ca}^{2+}\right]_{\mathrm{o}}$ at $2 \mathrm{~mm}$ and $\left[\mathrm{Mg}^{2+}\right]_{\mathrm{o}}$ at $1 \mathrm{mM}$. Electrical stimulation of the striatum produced a response that was confirmed as an eIPSC as a result of its complete sensitivity to bicuculline $(10 \mu \mathrm{M} ; n=3)$ (data not shown). eIPSCs were presumed monosynaptic because of their characteristic fixed latency $(0.21 \pm 0.02$ ms jitter; $n=7)$ (Fig. $2 \mathrm{~A}$ ) and that they were recorded under glutamatergic blockade. Because of the $\mathrm{CsCl}$ pipette solution, eIPSCs were inward currents at $-70 \mathrm{mV}$ and had a measured reversal potential of $5 \pm$ $4 \mathrm{mV}(n=3)$. Pairs of stimuli delivered at $50 \mathrm{~Hz}$ revealed that striatonigral eIPSCs underwent profound paired-pulse facilitation $(\mathrm{PPF})$, with an average paired-pulse ratio of $2.3 \pm 0.2(n=$ 23; range of $0.9-4.3$ ) (Fig. $2 B$ ). Because these recordings were made from juvenile animals [postnatal day 14 (P14) to P20] and short-term synaptic plasticity is known to change considerably as animals age (Mori-Kawakami et al., 2003; Fedchyshyn and Wang, 2005), we sought to track this PPF to confirm that it was not solely a feature of immature animals.
We recorded striatonigral eIPSCs from animals at 14, 15, 17, and $20 \mathrm{~d}$ old, at frequencies of 10, 50, and $100 \mathrm{~Hz}$ (Fig. 3). Frequency had no effect on the paired-pulse ratio $\left(F_{(2,55)}=0.08, p=\right.$ $0.9)$, but, as the animals aged, the paired-pulse ratio decreased $(\mathrm{P} 14,3.0 \pm 0.3 ; \mathrm{P} 15,2.5 \pm 0.3 ; \mathrm{P} 17,1.8 \pm 0.3 ; \mathrm{P} 20,2.0 \pm 0.2$; $\left.F_{(3,55)}=3.45, p=0.005\right)$. Applying a 10 pulse train revealed another effect: in young animals, PPF declined over many pulses at high frequencies. When comparing the ratio of the amplitude of the last event in the train to the first, at P14 there was marked potentiation at $10 \mathrm{~Hz}$, but at $100 \mathrm{~Hz}$ the last pulse was approaching the amplitude of the first $(\mathrm{P} 10 / \mathrm{P} 1: 10 \mathrm{~Hz}, 5.7 \pm$ $\left.1.9 ; 50 \mathrm{~Hz}, 4.0 \pm 0.3 ; 100 \mathrm{~Hz}, 1.7 \pm 0.4 ; F_{(2,13)}=5.3, p=0.02\right)$. However, by P20, there was no sign of the loss of PPF (P10/P1: $10 \mathrm{~Hz}, 3.6 \pm 1.3 ; 50 \mathrm{~Hz}, 4.5 \pm 1.8 ; 100 \mathrm{~Hz}, 3.7 \pm 1.7 ; F_{(2,16)}=$ $0.1, p=0.9)$. We noted that, although the paired-pulse ratio and the ratio of the first pulse to the last pulse changed over age, they had stabilized at P17, by which time they appeared to reach a stable phenotype.

We then investigated the effect of development on the rate of recovery from facilitation (Fig. 4). A burst of five pulses at $100 \mathrm{~Hz}$ was delivered to induce facilitation, followed by a single pulse delivered at $60,160,560,3000$, or $9000 \mathrm{~ms}$ after the last pulse in the train. Recovery could be fitted with an exponential, which at P14 had a time constant of $6.0 \pm 0.7 \mathrm{~s}$. The speed of recovery increased significantly over age, reaching a stable level of $2.0 \pm 0.7 \mathrm{~s}$ by P20 (P15, $2.7 \pm 0.4 \mathrm{~s} ; \mathrm{P} 17$, $\left.2.0 \pm 1.3 \mathrm{~s} ; F_{(3,19)}=3.8, p=0.03\right)$. 
A
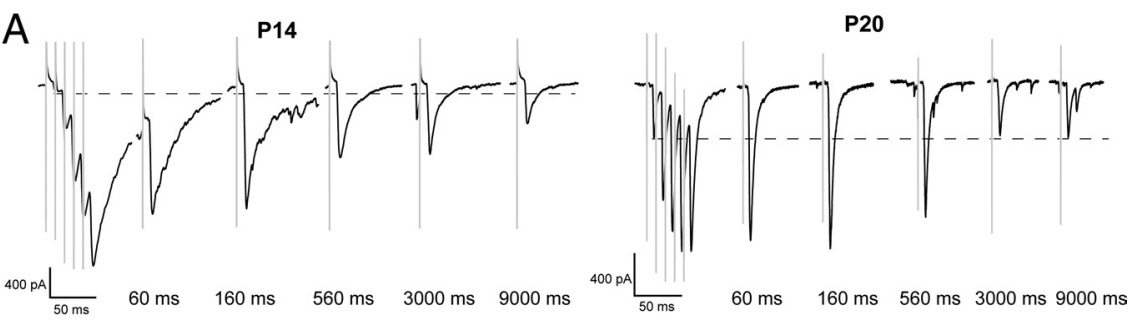

$\mathrm{B}$

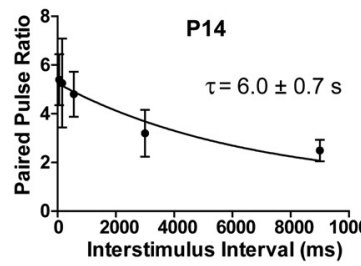

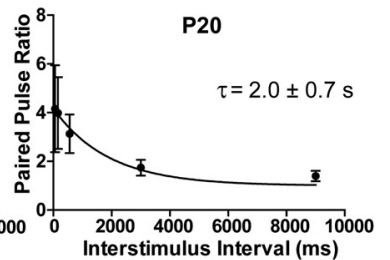

Interstimulus Interval (ms)
C

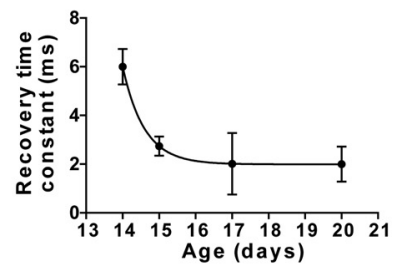

Figure 4. The speed of recovery from paired-pulse facilitation increases over development at striatonigral synapses. $A$, Waveforms showing the rate of recovery in P14 and P20 animals. Dotted line shows the amplitude of the first event. $B$, The recovery from facilitation in P14 $(n=4)$ and P20 $(n=6)$ proceeds in an exponential manner. $C$, Group statistics show changes in the rate of recovery over development $(n=4-6 ; p=0.03)$.

A
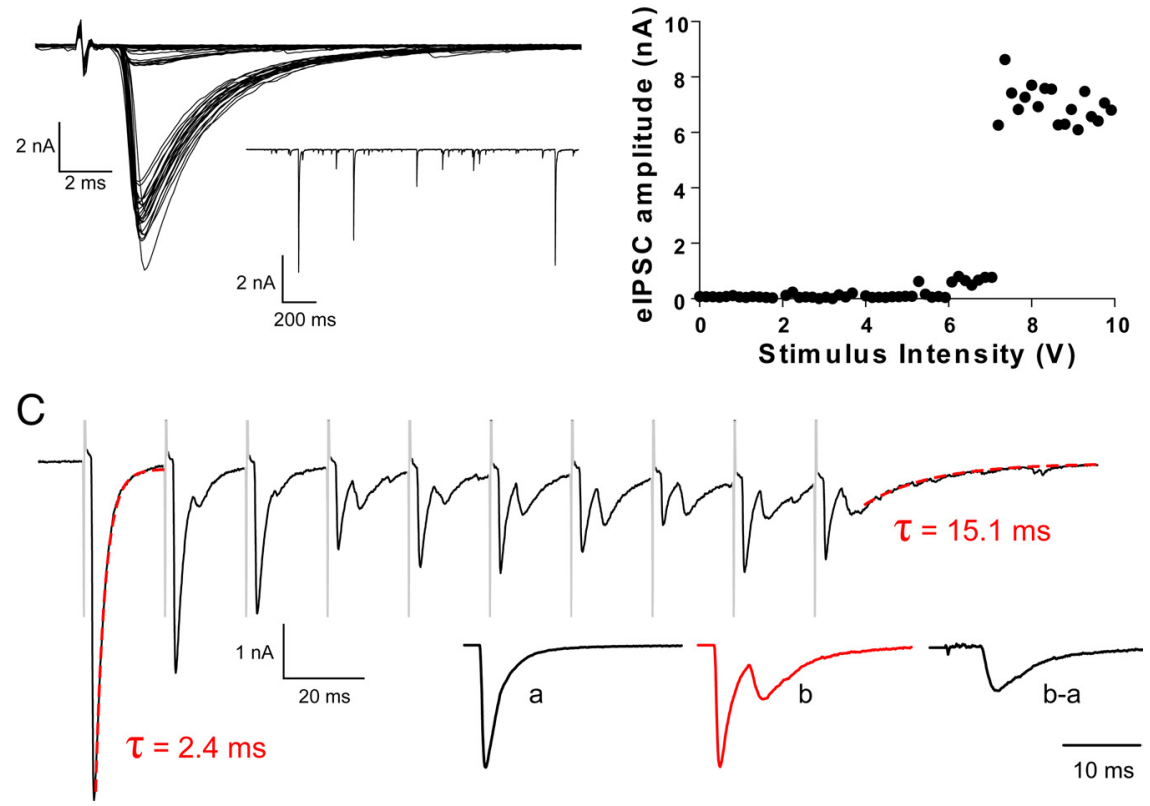

Figure 5. Minimal stimulation used to activate pallidonigral neurons. $\boldsymbol{A}$, Steadily increasing stimulus intensity recruited two discernable levels of IPSC amplitude. Inset, Typical 2-s-long trace of data showing spontaneous IPSCS with amplitudes $>5 \mathrm{nA}$. $\boldsymbol{B}$, Relationship between stimulus intensity and eIPSC amplitude from the cell shown in A. C, Occasionally, minimal stimulation in the GP evoked more than a single synaptic event. A fast, presumably pallidal, event can be seen depressing during a train, whereas a second slow, presumably striatal, event can be seen facilitating. Inset, $\boldsymbol{a}$, The first event in a train in which mixed events were evoked; $\boldsymbol{b}$, the last event in the train, scaled to the peak amplitude of the first. The first event, subtracted from last, reveals a synaptic event with a long latency, slow rise time, and slow decay time, characteristic of a striatonigral event.

As well as receiving dendritic inhibition from striatal medium spiny cells, nigral neurons are somatically targeted by projections from the GP. We sought to selectively activate these cells (and avoid striatonigral axons) by using a minimal stimulation paradigm (Fig. 5). Responses elicited by pallidal stimulation were completely blocked by bicuculline ( $10 \mu \mathrm{M} ; n=3)$ (data not shown), confirming their identity as eIPSCs. The eIPSC was often surprisingly large (mean, $5.3 \pm 0.6 \mathrm{nA}$; range, $0.61-24.9 \mathrm{nA}$ ), suggesting that there may have been more than one axon re- cruited by our minimal stimulation paradigm. However, spontaneous IPSCs with kinetics matching those of pallidonigral eIPSCs (rise time, $>0.6 \mathrm{~ms}$; decay constant, $>3 \mathrm{~ms}$; see below) were regularly recorded with amplitudes of $>5 \mathrm{nA}$ (Fig. $5 A$, inset). This indicates that there are single pallidonigral axons capable of producing IPSCs of this magnitude.

Even with minimal stimulation, occasionally a biphasic IPSC was evoked (Fig. $5 C$ ). Three things were noted about the second phase of these events. First, it invariably showed paired-pulse facilitation. By digitally subtracting the first (pallidonigral) event from a scaled biphasic trace, we noted the slow kinetics of the second phase, matching striatonigral events (decay time constant, $9.1 \pm 1.0 \mathrm{~ms} ; 10-90 \%$ rise time, $1.2 \pm 0.1 \mathrm{~ms} ; n=4$; see below). Last, the longer latency of the second phase of the event could be clearly seen $(5.1 \pm 0.2 \mathrm{~ms} ; n=4)$. This gives a conduction velocity of $0.68 \mathrm{~m} / \mathrm{s}$, matching striatonigral fibers (see below). Recordings in which this biphasic event occurred were excluded from additional analysis.

Additional experimental analyses highlighted significant differences between IPSCs evoked from striatum and GP. First, pallidonigral eIPSC exhibited significantly faster kinetics (Fig. 6). At P14, striatonigral IPSCs decayed with a time constant of $10.2 \pm 1.1 \mathrm{~ms}$, which decreased significantly over development to $5.2 \pm 0.4 \mathrm{~ms}$ by P20. Conversely, pallidonigral IPSCs decayed with a time constant of $2.5 \pm 0.1 \mathrm{~ms}$ at P14, which had decreased by P20 to $2.1 \pm 0.1 \mathrm{~ms}$ (age effect, $F_{(3,56)}=10.1, p<0.0001$; pathway effect, $F_{(1,56)}=204.4, p<0.0001$ ). Likewise, striatonigral IPSCs had a significantly slower $10-90 \%$ rise time, which also decreased over age (striatonigral: P14, $1.5 \pm$ $0.1 \mathrm{~ms} ; \mathrm{P} 15,1.3 \pm 0.1 \mathrm{~ms} ; \mathrm{P} 17,1.3 \pm 0.1 \mathrm{~ms}$; P20, $1.2 \pm 0.1 \mathrm{~ms}$; pallidonigral: P14, $0.49 \pm$ 0.06 ms; P15, $0.49 \pm 0.03$ ms; P17, $0.42 \pm$ $0.03 \mathrm{~ms} ; \mathrm{P} 20,0.41 \pm 0.02 \mathrm{~s} ;$ age effect, $F_{(3,56)}$ $=3, p=0.04$; pathway effect, $F_{(1,56)}=355$, $p<0.0001$ ) (data not shown). There was no change in input resistance during this phase of development (P14, $146 \pm 14 \mathrm{M} \Omega$; P15, $144 \pm 11 \mathrm{M} \Omega ; \mathrm{P} 17,154 \pm 10 \mathrm{M} \Omega ; \mathrm{P} 20$, $\left.144 \pm 20 \mathrm{M} \Omega ; F_{(3,34)}=0.1, p=0.9\right)($ data not shown). There was also a notable reduction in conduction delay over development, apparent from the decreasing response latencies of striatonigral and pallidonigral eIPSCs with age. At P14, the average conduction delay for striatonigral traffic was $8.6 \pm 0.2 \mathrm{~ms}(n=7)$, and by P20 this had dropped to $6.1 \pm$ $0.4 \mathrm{~ms}(n=5)(\mathrm{P} 15,8.0 \pm 0.2 \mathrm{~ms}$; P17, $7.2 \pm 0.1 \mathrm{~ms})$. Likewise, the conduction delay of pallidonigral eIPSCs dropped from $2.2 \pm 0.1 \mathrm{~ms}$ $(n=5)$ to $1.5 \pm 0.1 \mathrm{~ms}(n=9)(\mathrm{P} 15,2.0 \pm 0.1 \mathrm{~ms} ; \mathrm{P} 17,1.9 \pm 0.0 \mathrm{~ms}$; age effect, $F_{(3,45)}=35.1, p<0.0001$; pathway effect, $F_{(1,45)}=2203$, $p<0.0001)$. Given that the straight line distance from the striatal 
stimulating electrode to the $\mathrm{SNr}$ was $\sim 4.5$ $\mathrm{mm}$ whereas the distance from the pallidal stimulating distance to $\mathrm{SNr}$ was $\sim 3.5 \mathrm{~mm}$, this indicates that the conduction velocity for medium spiny axons increases from $0.5 \mathrm{~m} / \mathrm{s}$ at P14 to $0.7 \mathrm{~m} / \mathrm{s}$ by P20. Pallidonigral axons, conversely, conduct at $1.6 \mathrm{~m} / \mathrm{s}$ at $\mathrm{P} 14$, and this increases to $2.3 \mathrm{~m} / \mathrm{s}$ by P20.

The most striking difference between pallidonigral and striatonigral IPSCs, however, was that pallidonigral events showed paired-pulse depression (PPD), with an average paired-pulse ratio of $0.67 \pm 0.04(n=25$; range, $1.1-0.04)$. Again, we tracked the paired-pulse ratio over frequency and development but found these had no effect on the paired-pulse ratio (age effect, $F_{(3,59)}=1.3, p=0.3$; frequency effect, $F_{(2,59)}=0.8, p=0.4$ ) (Fig. $7 A-C$ ). Assuming that vesicle release follows a Poisson distribution, then the reciprocal of the square of the coefficient of variation of the amplitude of numerous evoked events $\left(1 / \mathrm{CV}^{2}\right)$ is directly proportional to the probability of release. Furthermore, even if release needs to be modeled by a more widely applicable binomial distribution, changes in $1 / \mathrm{CV}^{2}$ would equate to changes in the probability of release (Del Castillo and Katz, 1954). Because of this fact, we were able to investigate whether the paired-pulse depression of pallidonigral IPSCs was attributable to presynaptic (changes in the probability of release) or postsynaptic (changes in the quantal amplitude, i.e., receptor desensitization) mechanisms. Over five pulses at $100 \mathrm{~Hz}$, $1 / \mathrm{CV}^{2}$ decreased by $52 \pm 19 \%(n=3)$, whereas eIPSC amplitude decreased by $54 \pm 10 \%$ (Fig. $7 D-F)$. Therefore, PPD at pallidonigral terminals can be explained solely by a decrease in the probability of release. Furthermore, in contrast to the speed of recovery from PPF at striatonigral synapses that increased with age, we found that that the speed of recovery of PPD at pallidonigral terminals slowed over development. Recovery in P14 animals exhibited a time constant of $600 \pm 150 \mathrm{~ms}(n=4)$, slowing by P20 to $1760 \pm 230 \mathrm{~ms}(n=$ 9; $\left.\mathrm{P} 15,1330 \pm 260 \mathrm{~ms} ; \mathrm{P} 17,1780 \pm 210 \mathrm{~ms} ; F_{(3,21)}=4.2, p=0.02\right)$ (Fig. 8). Again, a stable phenotype was apparent by P17.

To investigate the functional consequences of the differing kinetic and short-term dynamics between striatal and pallidal inputs, we made current-clamp recordings in nigral neurons from P17-P21 animals. We used a pipette solution that contained $9.5 \mathrm{mM} \mathrm{Cl}^{-}$to mimic that measured by gramicidin perforated patch recordings (Gulácsi et al., 2003). For pallidal input, this gave a measured reversal potential of $-72 \pm 2 \mathrm{mV}(n=5)$, whereas for striatal input, it was $-80 \pm 3 \mathrm{mV}(n=5 ; p<0.05)$. We assume that this difference was attributable to the differing electrotonic distances of striatonigral and pallidonigral synapses to the recording electrode, but we cannot rule out a contribution of $\mathrm{Cl}^{-}$regulator mechanisms. We were curious about whether temporal summation of pallidal IPSPs could overcome synaptic depression. When current was injected to hold the membrane potential at approximately $-60 \mathrm{mV}$ and arrest pacemaker activity, striatal IPSPs still clearly facilitated and pallidal IPSPs still clearly depressed (Fig. 9A,B). We stimulated either the pallidonigral (minimally) or striatonigral pathways for $1 \mathrm{~s}$ at $100 \mathrm{~Hz}$. Pallidal events had a mean resting conductance of $20 \mathrm{nS}$ (range, 10.7-35 nS) versus $3 \mathrm{nS}$ (range, 2.1-3.8 nS) for striatal events. Although pallidal inputs were able to initially silence nigral neurons in four of four cells, three cells began firing while the pallidal events were still being evoked. Con- versely, in four of four cells, striatal events were able to silence nigral activity for the full second.

\section{Discussion}

Here, we clearly show differences in the effect of two converging inhibitory pathways on neurons in the major output nucleus of the basal ganglia. We discovered that striatonigral GABA release facilitates strongly, whereas pallidonigral synapses depress. We found that pallidonigral IPSCs are considerably faster than striatonigral IPSCs. Our results show that striatonigral IPSCs recover from facilitation more rapidly, and pallidonigral IPSCs recover from depression more slowly, as the animals age, reaching a stable and potentially adult phenotype at $\sim 17 \mathrm{~d}$ old. Finally, we show that bursts of activity from single pallidal inputs are able to transiently silence the firing of nigral neurons. However, during prolonged activity, synaptic depression causes pallidal input to be less effective and allows nigral neurons to begin firing again. Conversely, striatonigral input can consistently silence nigral activity.

To understand the functional significance of our findings, one needs to consider the firing properties of striatal and pallidal neurons. Striatal cells fire at a low basal rate, typically $0.02-3 \mathrm{~Hz}$ (which may be an overestimate because studies often exclude silent cells), but, in response to movement, neurons may burst at up to $100 \mathrm{~Hz}$, for as long as $500 \mathrm{~ms}$ (Wilson and Groves, 1981; Ryan et al., 1989; Kimura, 1990; Mahon et al., 2006). Our results show that, if a burst of $10-100 \mathrm{~Hz}$ is preceded by a pause in firing of $>2 \mathrm{~s}$ (i.e., the time constant for the recovery from facilitation), then the burst will produce a profound enhancement of GABA release at striatonigral terminals. Thus, paired-pulse facilitation can be seen as an amplification, or high-pass filter mechanism: low levels of basal firing will produce only a small degree of GABA release, but high-frequency bursts will produce an enhanced release of GABA. Conversely, neurons in the GP are highly active, firing tonically at $50-70 \mathrm{~Hz}$, and these cells show movementrelated pauses (DeLong, 1971; Raz et al., 2000). At pallidonigral synapses, synaptic depression works to enhance the functional "contrast" between the pause and the reinitiation of tonic firing. During tonic firing, depression will work to decrease the release of GABA at pallidonigral synapses; however, during a sufficiently long pause, the synapse will recover from depression, inducing maximal GABA release during the reinitiation of tonic firing. Our current-clamp recordings show that a single pallidal neuron can have a powerful inhibitory influence on the firing of a nigral neuron, but during synaptic depression, this effect wanes. So, the effect of pallidal inhibition is maximal immediately after a movement-related pause in tonic firing. 

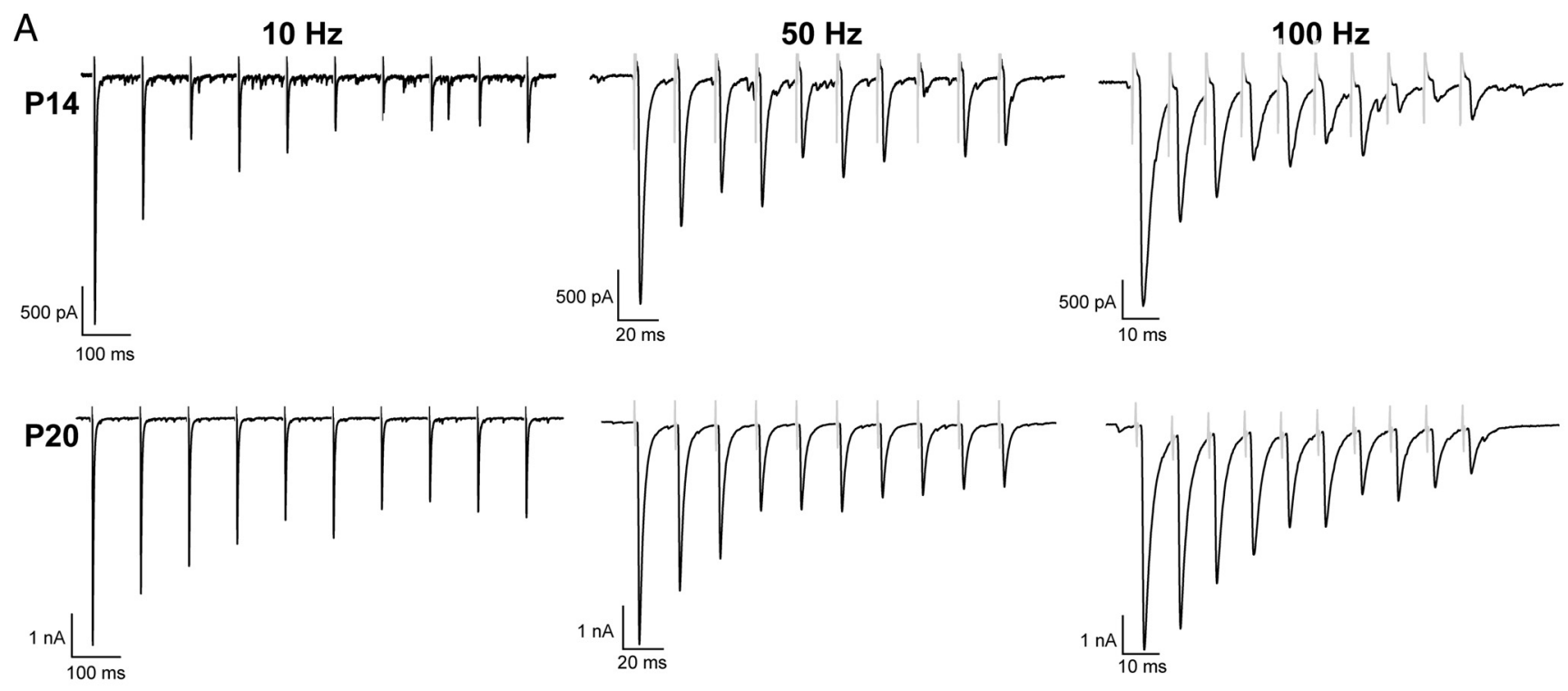

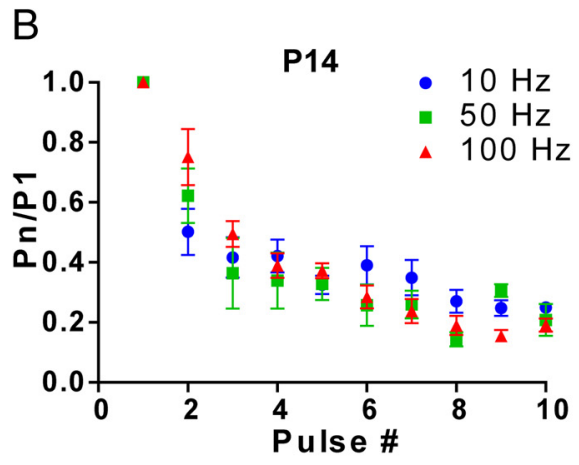

D

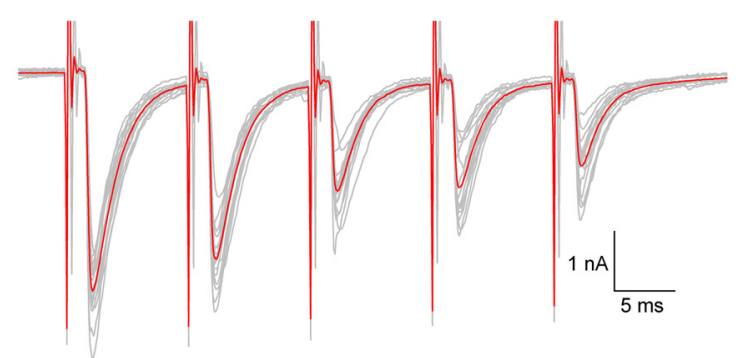

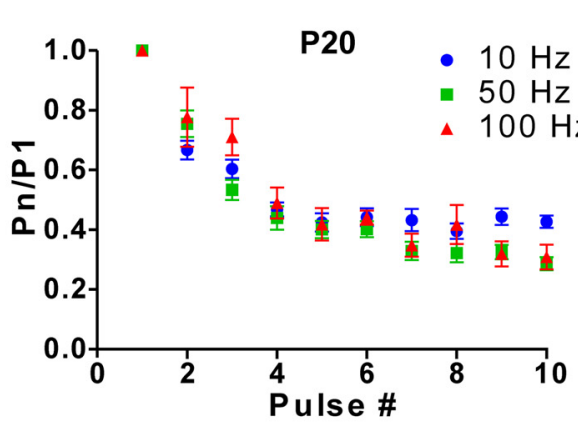

C

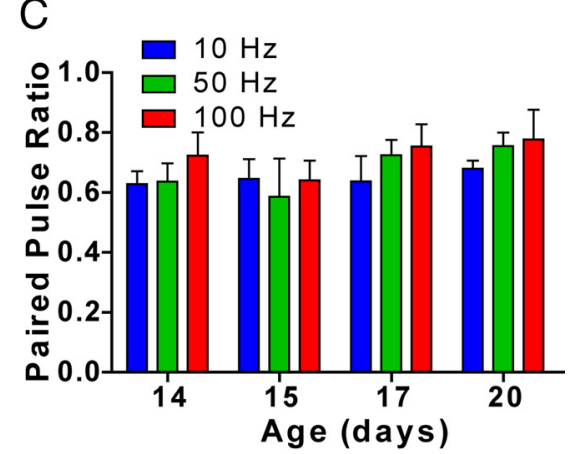

E

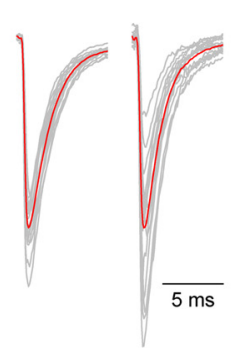

F

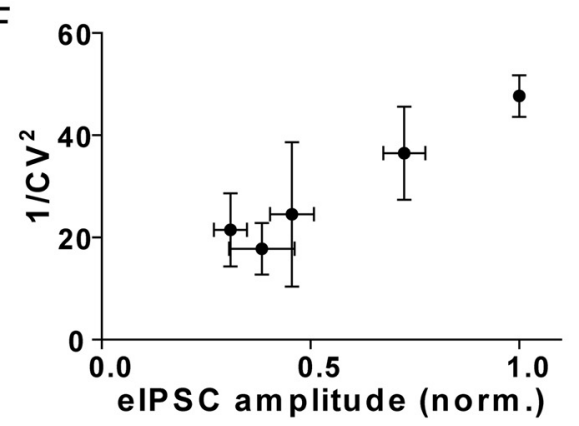

Figure 7. Pallidonigral elPSCs show paired-pulse depression. $\boldsymbol{A}$, Pallidonigral elPSCs evoked at 10, 50 , and $100 \mathrm{~Hz}$, at P14 and P20 showing clear paired-pulse depression. $\boldsymbol{B}$, At P14 ( $n=5-6)$ and P20 ( $n=7-8)$, there is no effect of frequency of PPD. C, Group statistics showing the lack of effect of frequency or age on the paired-pulse ratio $(n=4-8 ; p>0.3)$. D, Overlay of a five pulse train at $50 \mathrm{~Hz}$. Calibration: $1 \mathrm{nA}, 5 \mathrm{~ms}$. $\boldsymbol{E}$, The first and last pulse in the train scaled to the same amplitude to show the increase in the coefficient of variation. Calibration: $5 \mathrm{~ms}$. $\boldsymbol{F}, 1 / \mathrm{CV}^{2} \mathrm{decreases}$ by $\sim 50 \%$, the same degree as the amplitude of the synaptic events $(n=3)$, indicating that PPD is solely attributable to a presynaptic mechanism.

There have been numerous reports of the kinetics of GABAergic events increasing over development (Hollrigel and Soltesz, 1997; Huntsman and Huguenard, 2000; Okada et al., 2000). Consistent with our findings for evoked responses in SNr neurons, recently Chudomel et al. (2009) reported that the kinetics of spontaneous IPSCs in nigral neurons increase with age. Furthermore, the authors report an exchange of $\alpha 3$ subunits at perisomatic $\mathrm{GABA}_{\mathrm{A}}$ receptors with $\alpha 1$ subunits. Because pallidonigral synapses form somatically, this likely explains their enhanced kinetics with development because $\alpha 1$-containing $\mathrm{GABA}_{\mathrm{A}}$ receptors are known to have a fast deactivation rate (Gingrich et al., 1995; Picton and Fisher, 2007). The functional reason for the change in kinetics of striatonigral events is unclear. Because stria- tonigral events are likely generated at distal dendritic sites, their kinetics will be further affected by the cable properties of nigral cell dendrites. Indeed, it is unclear whether the difference in kinetics between striatal and pallidal events is solely attributable to cable filtering. Although we found that the input resistance of $\mathrm{SNr}$ cells does not change with development, this was measured at the soma and is unlikely to measure a change in dendritic membrane resistance. Therefore, it is unclear whether the changes in striatonigral IPSC kinetics is attributable to changes in $\mathrm{GABA}_{\mathrm{A}}$ receptor properties or are attributable to changes in dendritic membrane properties.

We also found that conduction velocity increases with development. This is likely to be attributable to the increase in myeli- 
nation seen in the CNS throughout (Hamano et al., 1998; Salami et al., 2003). Interestingly, this was the one property we measured that had not reached a stable phenotype by P17. Indeed, myelination of the internal capsule [which pallidonigral and striatonigral fibers pass through in rodents (Totterdell et al., 1984; Gustafson et al., 1989)] does not reach maturity until P42 (Hamano et al., 1998). By measuring the straight line distance between the stimulating electrode and the $\mathrm{SNr}$, we calculated that the conduction velocity of medium spiny axons was $0.7 \mathrm{~m} / \mathrm{s}$, in excellent agreement Ryan et al. (1986), whereas pallidonigral axons conducted at $2.3 \mathrm{~m} / \mathrm{s}$. This large difference could be explained if striatonigral axons were considerably tortuous, such that the linear distance significantly underestimated the true axonal length. However, anatomical evidence strongly argues against this idea (Kawaguchi et al., 1990; Wu et al., 2000). A parsimonious explanation is that pallidonigral axons become myelinated as soon as they enter the internal capsule and remain so throughout their journey to $\mathrm{SNr}$ (Smith and Bolam, 1989), whereas the small-caliber striatonigral axons are only thinly and sparsely myelinated (Somogyi and Smith, 1979; Chang et al., 1981).

We also reported that minimal stimulation in the globus pallidus produced very large synaptic events $(5.3 \mathrm{nA}, \sim 76 \mathrm{nS})$. This is in line with the numerous, large synaptic boutons formed by pallidonigral axons (Smith and Bolam, 1989). It would be useful to know the size of unitary striatonigral connections, but we were unable to record clear steps in amplitude during minimal stimulation in the striatum.

When considered from a whole circuit standpoint, these findings have two primary implications. First, tonic firing of pallidal neurons should have little effect on the activity of $\mathrm{SNr}$ neurons, because synaptic depression will quickly reduce the amount of GABA being released at pallidonigral synapses. Pallidal neurons will need to pause before they can transmit information to the SNr. Indeed, the amplitude of this signal will depend primarily on the length of the preceding pause. Thus, the GP may inform the $\mathrm{SNr}$ about the magnitude of the inhibition it has just received. Second, the facilitation seen at striatonigral synapses allows for nonlinear information transfer through the basal ganglia. Weak cortical activation, capable of inducing a single action potential in a population of medium spiny neurons, will have only minimal impact on nigral neurons. However, as corticostriatal traffic increases and medium spiny neurons are driven to burst, the inhibition they will deliver to the $\mathrm{SNr}$ increases significantly (approximately linearly with each subsequent action potential), meaning that
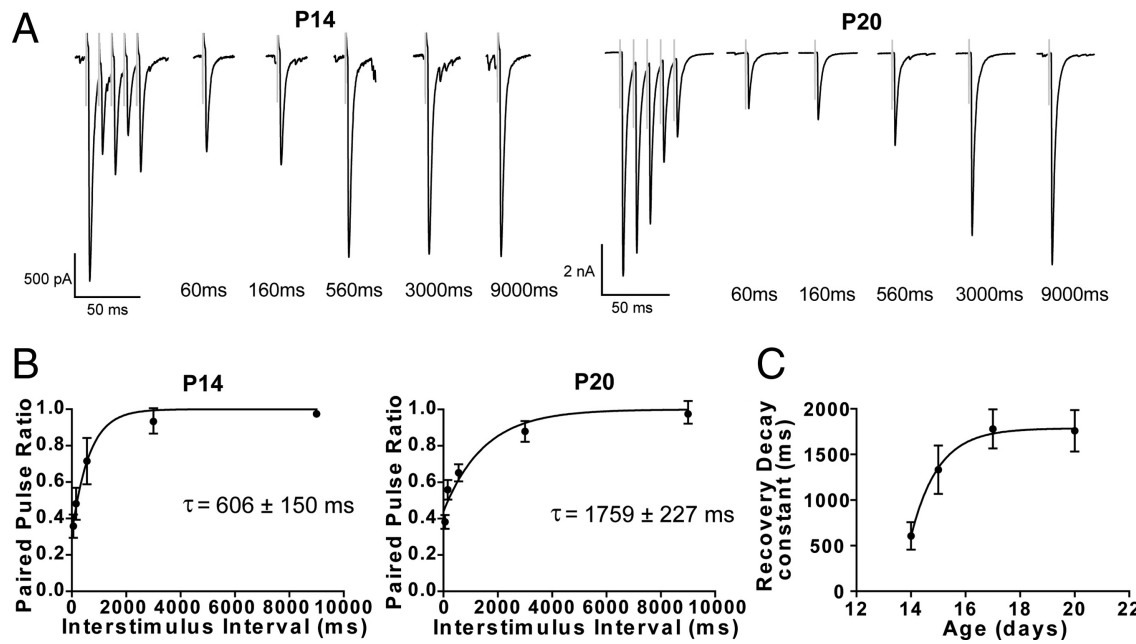

Figure 8. The recovery from paired-pulse depression at pallidonigral synapses slows over development. $\boldsymbol{A}$, Example traces showing the recovery from PPD at P14 and P20. $B$, The recovery from PPD at P14 $(n=4)$ and P20 $(n=9)$ can be fit with an exponential. $C$, The time constant of the recovery process significantly increases over age $(n=4-9 ; p=0.02)$.
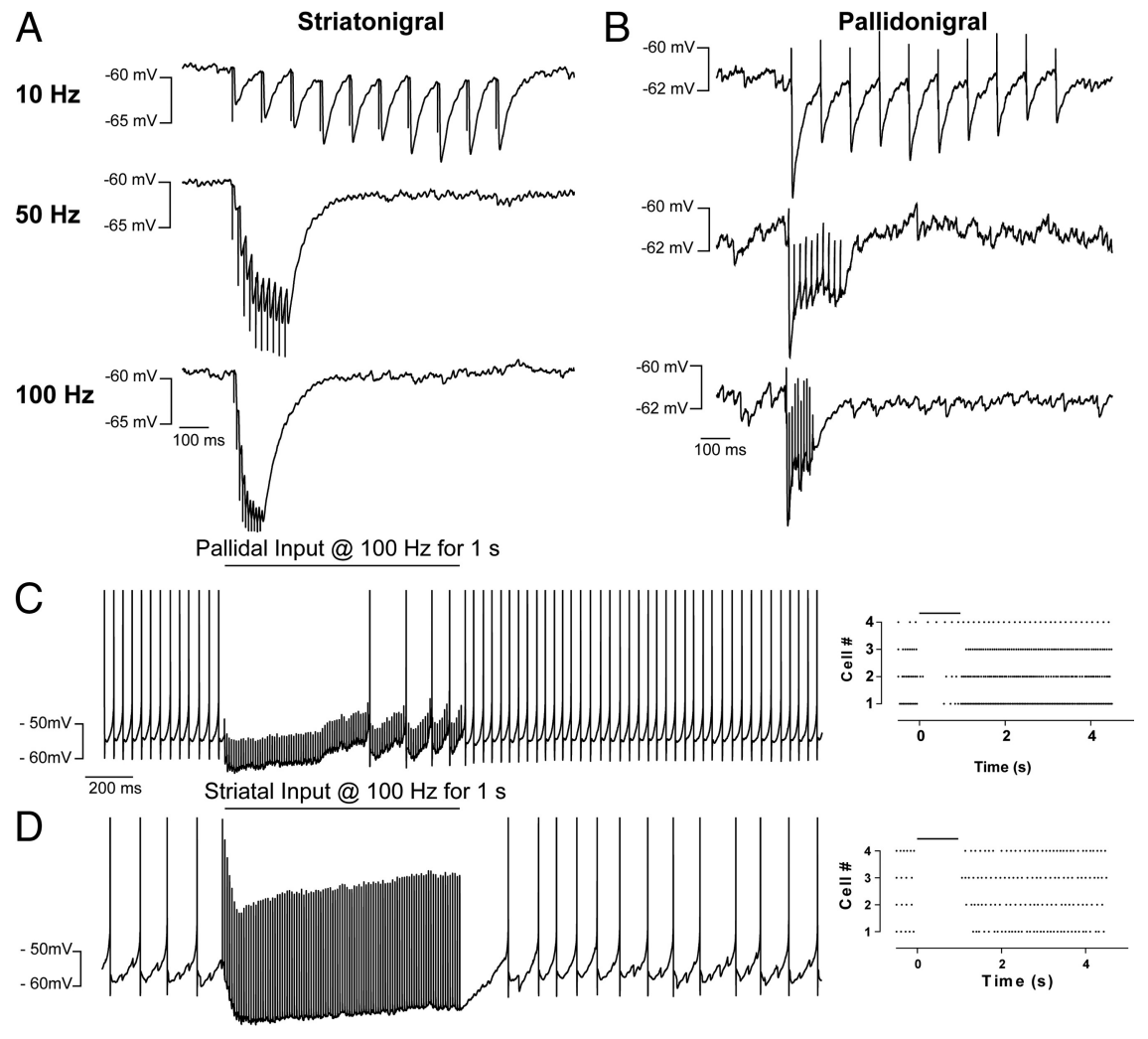

Figure 9. Striatonigral and pallidonigral input differentially regulate the pacemaker activity of nigral neurons. When $\mathrm{SNr}$ neurons are hyperpolarized, striatonigral inputs produce facilitating IPSPs $(\boldsymbol{A})$ whereas pallidonigral IPSPs depress $(\boldsymbol{B})$, even when they summate. C, During $1 \mathrm{~s}, 100 \mathrm{~Hz}$ trains, pallidal inputs initially silence nigral neurons, but in three of four cells, nigral neurons begin firing during the train. Left, Peristimulus time histogram, showing the activity of four nigral neurons during the pallidal stimulus (black line). D, The $1 \mathrm{~s}, 100 \mathrm{~Hz}$ striatonigral trains silence nigral activity during the entire duration of the train.

striatal neurons cannot only signal the timing of cortical activation but also its strength. Furthermore, this seems to indicate that spatial summation of a large number of different, weakly activated medium spiny neurons will be less effective at silencing nigral activity than a small population of strongly activated striatal cells. Interestingly, extracellular recording studies in awake animals have shown that taskdependent activation is transferred from a broad population of 
striatal neurons to a small subpopulation that responds increasingly stronger during learning (Barnes et al., 2005). These converging influences from direct and indirect pathways will determine the dynamics of inhibition of tonic firing of $\mathrm{SNr}$ neurons and the resulting disinhibition of target nuclei in thalamus and brainstem leading to movement initiation (Chevalier and Deniau, 1990).

\section{References}

Araki M, McGeer PL, McGeer EG (1985) Striatonigral and pallidonigral pathways studied by a combination of retrograde horseradish peroxidase tracing and a pharmacohistochemical method for gamma-aminobutyric acid transaminase. Brain Res 331:17-24.

Barnes TD, Kubota Y, Hu D, Jin DZ, Graybiel AM (2005) Activity of striatal neurons reflects dynamic encoding and recoding of procedural memories. Nature 437:1158-1161.

Beurrier C, Ben-Ari Y, Hammond C (2006) Preservation of the direct and indirect pathways in an in vitro preparation of the mouse basal ganglia. Neuroscience 140:77-86.

Chang HT, Wilson CJ, Kitai ST (1981) Single neostriatal efferent axons in the globus pallidus: a light and electron microscopic study. Science 213:915-918.

Chevalier G, Deniau JM (1990) Disinhibition as a basic process in the expression of striatal functions. Trends Neurosci 13:277-280.

Chudomel O, Herman H, Nair K, Moshé SL, Galanopoulou AS (2009) Ageand gender-related differences in GABAA receptor-mediated postsynaptic currents in GABAergic neurons of the substantia nigra reticulata in the rat. Neuroscience 163:155-167.

Del Castillo J, Katz B (1954) Quantal components of the end-plate potential. J Physiol 124:560-573.

DeLong MR (1971) Activity of pallidal neurons during movement. J Neurophysiol 34:414-427.

Deniau JM, Kitai ST, Donoghue JP, Grofova I (1982) Neuronal interactions in the substantia nigra pars reticulata through axon collaterals of the projection neurons. An electrophysiological and morphological study. Exp Brain Res 47:105-113.

Fedchyshyn MJ, Wang LY (2005) Developmental transformation of the release modality at the calyx of Held synapse. J Neurosci 25:4131-4140.

German DC, Manaye KF (1993) Midbrain dopaminergic neurons (nuclei A8, A9, and A10): three-dimensional reconstruction in the rat. J Comp Neurol 331:297-309.

Gingrich KJ, Roberts WA, Kass RS (1995) Dependence of the GABAA receptor gating kinetics on the alpha-subunit isoform: implications for structurefunction relations and synaptic transmission. J Physiol 489:529-543.

Gulácsi A, Lee CR, Sík A, Viitanen T, Kaila K, Tepper JM, Freund TF (2003) Cell type-specific differences in chloride-regulatory mechanisms and $\mathrm{GABA}_{\mathrm{A}}$ receptor-mediated inhibition in rat substantia nigra. J Neurosci 23:8237-8246.

Gustafson EL, Ouimet CC, Greengard P (1989) Spatial relationship of the striatonigral and mesostriatal pathways: double-label immunocytochemistry for DARPP-32 and tyrosine hydroxylase. Brain Res 491:297-306.

Hamano K, Takeya T, Iwasaki N, Nakayama J, Ohto T, Okada Y (1998) A quantitative study of the progress of myelination in the rat central nervous system, using the immunohistochemical method for proteolipid protein. Brain Res Dev Brain Res 108:287-293.

Hille B (2001) Selective permeability: independence. In: Ion channels of excitable membranes, Ed 3, pp 441-470. Sunderland, MA: Sinauer.

Hollrigel GS, Soltesz I (1997) Slow kinetics of miniature IPSCs during early postnatal development in granule cells of the dentate gyrus. J Neurosci 17:5119-5128.

Huntsman MM, Huguenard JR (2000) Nucleus-specific differences in $\mathrm{GABA}_{\mathrm{A}}$-receptor-mediated inhibition are enhanced during thalamic development. J Neurophysiol 83:350-358.

Kawaguchi Y, Wilson CJ, Emson PC (1990) Projection subtypes of rat neostriatal matrix cells revealed by intracellular injection of biocytin. J Neurosci 10:3421-3438.

Kimura M (1990) Behaviorally contingent property of movement-related activity of the primate putamen. J Neurophysiol 63:1277-1296.

Kita H, Kitai ST (1987) Efferent projections of the subthalamic nucleus in the rat: light and electron microscopic analysis with the PHA-L method. J Comp Neurol 260:435-452.

Kiyatkin EA, Rebec GV (1996) Dopaminergic modulation of glutamate- induced excitations of neurons in the neostriatum and nucleus accumbens of awake, unrestrained rats. J Neurophysiol 75:142-153.

Lavoie B, Parent A (1994) Pedunculopontine nucleus in the squirrel monkey: projections to the basal ganglia as revealed by anterograde tracttracing methods. J Comp Neurol 344:210-231.

Mahon S, Vautrelle N, Pezard L, Slaght SJ, Deniau JM, Chouvet G, Charpier S (2006) Distinct patterns of striatal medium spiny neuron activity during the natural sleep-wake cycle. J Neurosci 26:12587-12595.

Mori-Kawakami F, Kobayashi K, Takahashi T (2003) Developmental decrease in synaptic facilitation at the mouse hippocampal mossy fibre synapse. J Physiol 553:37-48.

Naito A, Kita H (1994) The cortico-nigral projection in the rat: an anterograde tracing study with biotinylated dextran amine. Brain Res 637:317-322.

Okada M, Onodera K, Van Renterghem C, Sieghart W, Takahashi T (2000) Functional correlation of $\mathrm{GABA}_{\mathrm{A}}$ receptor $\alpha$ subunits expression with the properties of IPSCs in the developing thalamus. J Neurosci 20:2202-2208.

Picton AJ, Fisher JL (2007) Effect of the alpha subunit subtype on the macroscopic kinetic properties of recombinant $\mathrm{GABA}_{\mathrm{A}}$ receptors. Brain Res 1165:40-49.

Preston RJ, Bishop GA, Kitai ST (1980) Medium spiny neuron projection from the rat striatum: an intracellular horseradish peroxidase study. Brain Res 183:253-263.

Raz A, Vaadia E, Bergman H (2000) Firing patterns and correlations of spontaneous discharge of pallidal neurons in the normal and the tremulous 1-methyl-4-phenyl-1,2,3,6-tetrahydropyridine vervet model of parkinsonism. J Neurosci 20:8559-8571.

Ribak CE, Vaughn JE, Roberts E (1980) GABAergic nerve terminals decrease in the substantia nigra following hemitransections of the striatonigral and pallidonigral pathways. Brain Res 192:413-420.

Richards CD, Shiroyama T, Kitai ST (1997) Electrophysiological and immunocytochemical characterization of GABA and dopamine neurons in the substantia nigra of the rat. Neuroscience 80:545-557.

Ryan LJ, Young SJ, Groves PM (1986) Substantia nigra stimulation evoked antidromic responses in rat neostriatum. Exp Brain Res 63:449-460.

Ryan LJ, Young SJ, Segal DS, Groves PM (1989) Antidromically identified striatonigral projection neurons in the chronically implanted behaving rat: relations of cell firing to amphetamine-induced behaviors. Behav Neurosci 103:3-14.

Salami M, Itami C, Tsumoto T, Kimura F (2003) Change of conduction velocity by regional myelination yields constant latency irrespective of distance between thalamus and cortex. Proc Natl Acad Sci U S A 100:6174-6179.

Smith Y, Bolam JP (1989) Neurons of the substantia nigra reticulata receive a dense GABA-containing input from the globus pallidus in the rat. Brain Res 493:160-167.

Smith Y, Bolam JP (1991) Convergence of synaptic inputs from the striatum and the globus pallidus onto identified nigrocollicular cells in the rat: a double anterograde labelling study. Neuroscience 44:45-73.

Somogyi P, Smith AD (1979) Projection of neostriatal spiny neurons to the substantia nigra. Application of a combined Golgi-staining and horseradish peroxidase transport procedure at both light and electron microscopic levels. Brain Res 178:3-15.

Totterdell S, Bolam JP, Smith AD (1984) Characterization of pallidonigral neurons in the rat by a combination of Golgi impregnation and retrograde transport of horseradish peroxidase: their monosynaptic input from the neostriatum. J Neurocytol 13:593-616.

von Krosigk M, Smith Y, Bolam JP, Smith AD (1992) Synaptic organization of GABAergic inputs from the striatum and the globus pallidus onto neurons in the substantia nigra and retrorubral field which project to the medullary reticular formation. Neuroscience 50:531-549.

Wallmichrath I, Szabo B (2002) Cannabinoids inhibit striatonigral GABAergic neurotransmission in the mouse. Neuroscience 113:671-682.

Wilson CJ, Groves PM (1981) Spontaneous firing patterns of identified spiny neurons in the rat neostriatum. Brain Res 220:67-80.

Wu Y, Richard S, Parent A (2000) The organization of the striatal output system: a single-cell juxtacellular labeling study in the rat. Neurosci Res $38: 49-62$.

Yung WH, Häusser MA, Jack JJ (1991) Electrophysiology of dopaminergic and non-dopaminergic neurones of the guinea-pig substantia nigra pars compacta in vitro. J Physiol 436:643-667.

Zucker RS, Regehr WG (2002) Short-term synaptic plasticity. Annu Rev Physiol 64:355-405. 